\title{
Low Frequency Oscillations Damping by UPFC with GAPOD and GADC-voltage regulator
}

\author{
Dakka. Obulesu', Dr. S.F. Kodad ${ }^{2}$, Dr. B.V Sankar Ram ${ }^{3}$ \\ ${ }^{1}$ Asso Professor, Department of Electrical \& Electronics Engg., VEMU.I.T.,P.Kothakota, Chittoor dist, Andhra \\ Pradesh-517325, India. \\ ${ }^{2}$ Principal, Krishna Murthy Institute of Technology and Engineering, Hyderabad, India. \\ ${ }^{3}$ Director, Admissions, J.N.T.U.H, Hyderabad, India.
}

\begin{abstract}
Low frequency oscillations(LFO) are inevitable characteristics of power system which affect the transmission line transfer capability and the system stability. In this paper, a new POD controller with DCvoltage regulator based on genetic algorithm is proposed for the UPFC for damping low frequency oscillations. The effectiveness of the proposed controller has been tested on a SMIB(double-line) power system in comparison with PSOMSF DC-voltage regulator under different operating conditions. The construction and implementation of this controller is fairly easy, which can be useful in real world power system.

Keywords : UPFC, Multi-Stage Fuzzy Controller (MSF), GAPOD (Genetic Algorithms based Power oscillation damping), PSO based MSF (PSOMSF), GA DC-voltage regulator.
\end{abstract}

\section{INTRODUCTION}

The large-scale power system interconnection is intended to make electric energy generation and transmission more economical and reliable. The economic aspect is manifested through the drastic reduction of spinning reserve or the standby generating capacity for maintenance or emergency use, from $25 \%$ or more of the total capacity a few decades ago to much less in the modern electric power systems. The reliability of the interconnected system is enhanced by virtue of the capability of transferring power readily from one area to other within the system. The large-scale power system interconnection caused many new dynamic power system problems to emerge which include the low-frequency oscillations of the interconnected large electric power systems. The low-frequency oscillations are due to the lack of damping of the mechanical mode of the system.

A better utilization of the existing power systems to increase their capacities and controllability by installing FACTS devices becomes imperative. Due to the present situation, there are two main aspects that should be considered in using FACTS devices. The first aspect is the flexible power system operation according to the power flow control capability of FACTS devices. The other aspect is the improvement of transient and steady-state stability of power systems. FACTS devices are the right equipment to meet these challenges.

The Unified Power Flow Controller (UPFC) is regarded as one of the most versatile devices in the FACTS device family which has the ability to control power flow in the transmission line, improve the transient stability, mitigate system oscillation and provide voltage support. It performs this through the control of the inphase voltage, quadrate voltage and shunts compensation due to its mains control strategy [1].

In [2] N. Tambey, M.L. Kothari suggested that the addition of a conventional supplementary controller to the UPFC is an effective solution to the problem. However, an industrial process, such as a power system, contains different kinds of uncertainties due to continuous load changes or parameters drift due to power systems highly nonlinear and stochastic operating nature. As a result, a fixed parameter controller based on the classical control theory such as PI or lead-lag controller is not certainly suitable for the UPFC damping control methods. Thus, it is required that a flexible controller be developed. In [3-6] authors P.K. Dash, S. Mishra, B C.

$\mathrm{Pal}$, et al. suggested Artificial neural networks method and robust control methodologies to cope with system uncertainties to enhance the system damping performance using the UPFC. However, the parameters adjustments of these controllers need some trial and error. Also, although using the robust control methods, the uncertainties are directly introduced to the synthesis, due to the large model order of power systems the order resulting controller will be very large in general, which is not feasible because of the computational economical difficulties in implementing.

The fuzzy controller has a number of distinguished advantages over the conventional one. It is not so sensitive to the variation of system structure, parameters and operation points and can be easily implemented in a large-scale nonlinear system. The most attractive feature is its capability of incorporating human knowledge to the controller with ease. This approach provides the FL systems better functionality, performance, adaptability, reliability and robustness. The most dynamic area of fuzzy systems research in the power systems has been the stability enhancement and assessment [7-8]. 
In [9-11] Some authors used FL-based damping control strategy for TCSC, UPFC and SVC in a multimachine power system. The damping control strategy employs non-optimal FL controllers. That is why the system's response settling time is unbearable. Dash et al. presented a fuzzy damping control system for series connected FACTS devices, e.g. TCSC, UPFC and TCPST to enhance power system stability.

The FL-based damping controller may exhibit lack of robustness due to its simplicity and the system's response for a wide incursion in the operating condition is anticipated to deteriorate. Khon and Lo used a fuzzy damping controller designed by micro Genetic Algorithm (GA) for TCSC and UPFC to improve powers system low frequency oscillations. The proposed method may not have enough robustness due to its simplicity against the different kinds of uncertainties and disturbances [12]. Mok et al. applied a GA-based Proportional-Integral (PI) type fuzzy controller for UPFC to enhance power system damping. Although, the fuzzy PI controller is simpler and more applicable to remove the steady state error, it is known to give poor performance in the system transient response [13].Power system is highly non-linear and stochastic in nature so the fixed parameter conventional supplementary controllers are not suitable for UPFC. Thus, it is required that a flexible controller be developed. Recently, fuzzy logic controllers are used for UPFC because it provides better functionality, performance, adaptability, reliability and robustness. In these controllers trial and error method is used for the formation of fuzzy sets.

In order to overcome the above drawbacks, in this work a new Multi Stage Fuzzy (MSF) PID controller with fuzzy switch for the UPFC DC-voltage controller is introduced to enhance the dynamic stability. The membership functions are formed using genetic algorithms. This is a form of behavior based on controller, where PD controller becomes active when certain conditions are met. The resulting structure is a controller using two-dimensional inference engines (rule base) to reasonably perform the task of a three-dimensional controller. The proposed method requires fewer resources to operate and its role in the system response is more apparent, i.e. it is easier to understand the effect of a two-dimensional controller than a three-dimensional one.

One of the essential and important steps toward the design of any successful fuzzy controllers is accurately constructing the membership functions. On the other hand, extraction of an appropriate set of membership functions from the expert may be tedious, time consuming and process specific. Thus, in order to reduce fuzzy system effort a modified GA is used for the optimum tuning of the membership functions in the proposed MSF controller, automatically.

Genetic algorithms are heuristic search optimization techniques inspired by natural evolution and has attractive features such as robustness, simplicity, etc. However, it cannot guarantee that the best solution will be found. In fact, sometimes it converges to local, rather than global optima. To overcome this drawback, a modified GA based on the hill climbing method is proposed in this paper to improve optimization synthesis such that the global optima are guaranteed and the speed of algorithms convergence is extremely improved, too.

\section{Modeling Of Snigle Machine Infinite Bus System Installed With Upfe}

Fig.1. Shows a Single machine infinite-bus (SMIB) system equipped with UPFC. The UPFC is installed in one of the two parallel transmission lines. This configuration comprising two parallel transmission lines, permits the control of real and reactive power flow through a line. The UPFC consists of an excitation Transformer (ET), a boosting transformer (BT), two three-phase GTO based voltage source converters (VSCs) and a DC link capacitor. The four input control signals to the UPFC are $m_{E}, m_{B}, \delta_{E}$ and $\delta_{B}$ where $m_{E}$ is the excitation amplitude modulation ration, $m_{B}$ is the boosting amplitude modulation ratio, $\delta_{E}$ is the excitation phase angle and $\delta_{B}$ is the boosting phase angle.

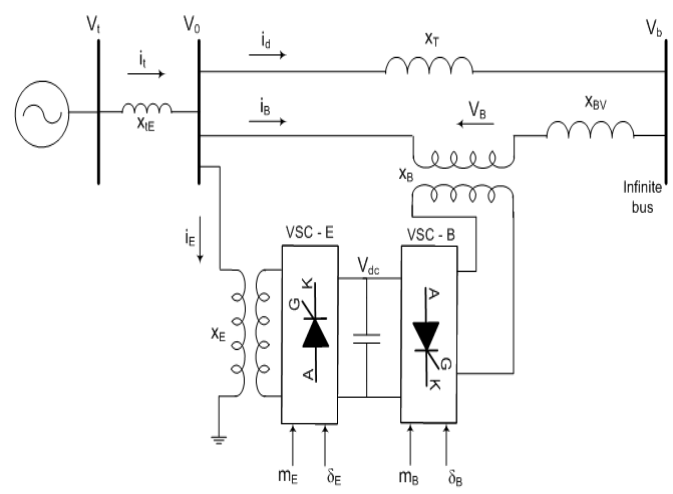

Fig.1 SMIB power system equipped with UPFC 
By applying Park's transformation and ignoring the resistance and transients of the ET and BT transformers ,the UPFC can be modeled as [14]

$$
\begin{aligned}
& {\left[\begin{array}{c}
V_{E t d} \\
V_{E t q}
\end{array}\right]=\left[\begin{array}{cc}
0 & -X_{E} \\
X_{E} & 0
\end{array}\right]\left[\begin{array}{l}
i_{E d} \\
i_{E q}
\end{array}\right]+\left[\begin{array}{l}
\frac{m_{E} \cos \left(\delta_{E}\right) V_{d c}}{2} \\
\frac{m_{E} \sin \left(\delta_{E}\right) V_{d c}}{2}
\end{array}\right]} \\
& {\left[\begin{array}{l}
V_{B t d} \\
V_{B t q}
\end{array}\right]=\left[\begin{array}{cc}
0 & -X_{B} \\
X_{B} & 0
\end{array}\right]\left[\begin{array}{l}
i_{B d} \\
i_{B q}
\end{array}\right]+\left[\begin{array}{l}
\frac{m_{B} \cos \left(\delta_{B}\right) V_{d c}}{2} \\
\frac{m_{B} \sin \left(\delta_{B}\right) V_{d c}}{2}
\end{array}\right]} \\
& \frac{d V_{d c}}{d t}=\frac{3 m_{E}}{4 C_{d c}}\left[\begin{array}{ll}
\cos \delta_{E} & \sin \delta_{E}
\end{array}\right]\left[\begin{array}{c}
i_{E d} \\
i_{E q}
\end{array}\right]+\frac{3 m_{B}}{4 C_{d c}}\left[\begin{array}{cc}
\cos \delta_{B} & \sin \delta_{B}
\end{array}\right]\left[\begin{array}{c}
i_{E d} \\
i_{E q}
\end{array}\right]
\end{aligned}
$$

Where $V_{E t}$ is the excitation voltage, $i_{E}$ is the excitation current, $V_{B t}$ is the boosting voltage and $i_{B}$ is the boosting current; $C_{d c}$ is the DC link capacitance and $V_{d c}$ is the DC link voltage. The nonlinear model of the SMIB system as shown in Fig.1 is described by [14].

$$
\begin{aligned}
& \dot{\omega}=\left(P_{m}-P_{e}-D \Delta \omega\right) / M \\
& \dot{\delta}=\omega_{o}(\omega-1) \\
& \dot{E}_{q}^{\prime}=\left(-E_{q}+E_{f d}\right) / T_{d o}^{\prime} \\
& \dot{E}_{f d}=\left(-E_{f d}+K_{a}\left(V_{r e f}-V_{t}\right)\right) / T_{a} \\
& V_{t}=V_{t d}+j V_{t q} ; \quad V_{t d}=X_{q} I_{t q} ; \quad V_{t q}=E_{q}^{\prime}-X_{d}^{\prime} I_{t d} \\
& I_{t d}=I_{t l d}+I_{E d}+I_{B d} ; \quad I_{t q}=I_{t l q}+I_{E q}+I_{B q} \\
& I_{t l d}=\frac{X_{E}}{X_{T}} I_{E d}+\frac{1}{X_{T}} \frac{m_{E} V_{d c} \cos \left(\delta_{E}\right)}{2}-\frac{1}{X_{T}} V_{b} \cos \delta \\
& I_{t l q}=\frac{X_{E}}{X_{T}} I_{E q}-\frac{1}{X_{T}} \frac{m_{E} V_{d c} \sin \left(\delta_{E}\right)}{2}+\frac{1}{X_{T}} V_{b} \sin \delta \\
& I_{E d}=\left[\frac{X_{d t}-X_{B B} X_{b 3}}{X_{d E}}\right] V_{b} \cos \delta-\left[\frac{X_{d t} m_{B} V_{d c} \cos \delta_{B}}{2 X_{d E}}\right]+\frac{X_{B B}}{X_{d E}} E_{q}^{\prime}-\left[\frac{X_{d t}+X_{B B} X_{b 2}}{X_{d E}}\right] \frac{m_{E} V_{d c} \cos \delta_{E}}{2} \\
& I_{E q}=\left[\frac{X_{d t}+X_{B B} X_{a 3}}{X_{q E}}\right] V_{b} \sin \delta-\left[\frac{X_{q t} m_{B} V_{d c} \sin \delta_{B}}{2 X_{q E}}\right]-\left[\frac{X_{q t}+X_{B B} X_{a 2}}{X_{q E}}\right] \frac{m_{E} V_{d c} \sin \delta_{E}}{2} \\
& I_{B d}=\left[\frac{X_{b 3} X_{E}-X_{b 1}}{X_{d E}}\right] V_{b} \cos \delta+\frac{X_{b 1} m_{B} V_{d c} \cos \delta_{B}}{2 X_{d E}}+\frac{X_{E}}{X_{d E}} E_{q}^{\prime}+\left[\frac{X_{b 1}-X_{E} X_{b 2}}{X_{d E}}\right] \frac{m_{E} V_{d c} \cos \delta_{E}}{2} \\
& I_{B q}=-\left[\frac{X_{a 3} X_{E}+X_{b 1}}{X_{q E}}\right] V_{b} \sin \delta+\frac{X_{a 1} m_{B} V_{d c} \sin \delta_{B}}{2 X_{q E}}+\left[\frac{X_{a 1}-X_{E} X_{a 2}}{X_{q E}}\right] \frac{m_{E} V_{d c} \sin \delta_{E}}{2} \\
& X_{d t}=X_{t E}+X_{d}^{\prime} ; X_{q t}=X_{q}+X_{t E} \quad X_{d s}=X_{E}+X_{d t} \quad ; \quad X_{q s}=X_{E}+X_{q t} \\
& X_{a 1}=\frac{\left(X_{q s} X_{T}+X_{q t} X_{E}\right)}{X_{T}} ; X_{b 1}=\frac{\left(X_{d s} X_{T}+X_{d t} X_{E}\right)}{X_{T}} \\
& X_{B B}=X_{B}+X_{B V} ; \quad X_{a 2}=1+\frac{X_{q t}}{X_{T}}
\end{aligned}
$$




$$
\begin{aligned}
& X_{b 2}=1+\frac{X_{d t}}{X_{T}} ; \quad X_{a 3}=-\frac{X_{q t}}{X_{T}} ; \quad X_{b 3}=\frac{X_{d t}}{X_{T}} \\
& X_{q E}=-\left(\frac{X_{B B} X_{q t} X_{E}}{X_{T}}+X_{E} X_{q t}+X_{B B} X_{q s}\right) \quad X_{d E}=\left(\frac{X_{B B} X_{d t} X_{E}}{X_{T}}+X_{E} X_{d t}+X_{B B} X_{d s}\right)
\end{aligned}
$$

The equation for real power balance between the series and shunt converters is given by:

$$
\operatorname{Re}\left(V_{B} I_{B}^{*}-V_{E} I_{E}^{*}\right)=0
$$

\section{A. Power system Linearized Dynamic Model}

The linear dynamic model is obtained by linearizing the nonlinear model around an operating condition. The linearized model of the power system as shown in Fig. 1 is given as follows:

$$
\begin{gathered}
\Delta \dot{\delta}=\omega_{o} \Delta \omega \\
\Delta \dot{\omega}=\frac{\Delta P_{m}-\Delta P_{e}-D \Delta \omega}{M} \\
\Delta \dot{E}_{f d}=-\frac{\Delta E_{f d}}{T_{A}}-\frac{K_{A} \Delta V}{T_{A}} \\
\Delta E_{q}=\left(X_{d}-X_{d}\right) \Delta i_{d}-\Delta E_{q}^{\prime} \\
\Delta \dot{V}_{d c}=K_{7} \Delta \delta+K_{8} \Delta E_{q}^{\prime}-K_{9} \Delta V_{d c}+K_{c e} \Delta m_{E}+K_{c \delta e} \Delta \delta_{E}+K_{c b} \Delta m_{B}+K_{c \delta b} \Delta \delta_{B}
\end{gathered}
$$

Where

$$
\begin{aligned}
& \Delta \dot{E}_{q}^{\prime}=\frac{-\Delta E_{q}+\Delta E_{f d}}{T_{d c}^{\prime}} ; \quad E_{q}=\left(X_{d}-X_{d}^{\prime}\right) i_{d}-E_{q}^{\prime} \quad ; \quad \Delta V=\Delta V_{r e f}-\Delta V_{t} \\
& \Delta P_{e}=K_{1} \Delta \delta+K_{2} \Delta E_{q}^{\prime}+K_{p d} \Delta V_{d c}+K_{p e} \Delta m_{E}+K_{p \delta e} \Delta \delta_{E}+K_{p b} \Delta m_{B}+K_{p \delta b} \Delta \delta_{B} \\
& \Delta E_{q}^{\prime}=K_{4} \Delta \delta+K_{3} \Delta E_{q}^{\prime}+K_{q d} \Delta V_{d c}+K_{q e} \Delta m_{E}+K_{q \delta e} \Delta \delta_{E}+K_{q b} \Delta m_{B}+K_{q \delta b} \Delta \delta_{B} \\
& \Delta V_{t}=K_{5} \Delta \delta+K_{6} \Delta E_{q}^{\prime}+K_{v d} \Delta V_{d c}+K_{v e} \Delta m_{E}+K_{v \delta e} \Delta \delta_{E}+K_{v b} \Delta m_{B}+K_{v \delta b} \Delta \delta_{B}
\end{aligned}
$$

$K_{1}, K_{2} \ldots K_{9}, K_{p u}, K_{q u}$ and $K_{v u}$ are the linearization constants. The SMIB power system state-space model is obtained from the linearized dynamic equations as:

$$
\dot{X}=A X+B U
$$

Where, the state vector $\mathrm{X}$, control vector $\mathrm{U}, \mathrm{A}$ and $\mathrm{B}$ are

$$
\begin{aligned}
& X=\left[\begin{array}{lllll}
\Delta \delta & \Delta \omega & \Delta E_{q}^{\prime} & \Delta E_{f d} & \Delta V_{d c}
\end{array}\right]^{T} \\
& U=\left[\begin{array}{lllll}
\Delta m_{E} & \Delta \delta_{E} & \Delta m_{B} & \Delta \delta_{B}
\end{array}\right]^{T} \\
& A= {\left[\begin{array}{ccccc}
0 & \omega_{0} & 0 & 0 & 0 \\
\frac{-K_{1}}{M} & 0 & \frac{-K_{2}}{M} & 0 & \frac{-K_{p d}}{M} \\
\frac{-K_{4}}{T_{d o}^{\prime}} & 0 & \frac{-K_{3}}{T_{d o}^{\prime}} & \frac{1}{T_{d o}^{\prime}} & \frac{-K_{q d}}{T_{d o}^{\prime}} \\
\frac{-K_{A} K_{5}}{T_{A}} & 0 & \frac{-K_{A} K_{6}}{T_{A}} & \frac{-1}{T_{A}} & \frac{-K_{A} K_{v d}}{T_{A}} \\
K_{7} & 0 & K_{8} & 0 & -K_{9}
\end{array}\right] } \\
& B=\left[\begin{array}{cccc}
\frac{-K_{p \delta e}}{M} & \frac{-K_{p b}}{M} & \frac{-K_{p \delta b}}{M} \\
\frac{-K_{p e}}{M} & \frac{-K_{q \delta e}}{T_{d o}^{\prime}} & \frac{-K_{q b}}{T_{d o}^{\prime}} & \frac{-K_{q \delta b}}{T_{d o}^{\prime}} \\
\frac{-K_{q e}^{\prime}}{T_{d o}^{\prime}} & \frac{-K_{A} K_{v \delta e}}{T_{A}} & \frac{-K_{A} K_{v b}}{T_{A}} & \frac{-K_{A} K_{v \delta b}}{T_{A}} \\
\frac{K_{c e}}{K_{c \delta e}} & \frac{K_{c b}}{K_{c \delta b}}
\end{array}\right]
\end{aligned}
$$




\section{UPFC Controllers Design}

To damp electromechanical oscillations in power system, supplementary control action can be applied to some FACTS devices to increase the system damping. The supplementary control is called power oscillation damping (POD). Since the FACTS devices are located in transmission systems, local input signals are always preferred, usually the active or reactive power flow through the FACTS device or the FACTS terminal voltages. POD control is applied very often on PSS. In that case the local rotor speed is the input signal for POD controller.

PI-type DC Voltage regulators shown in fig. 2 and Fig.3 shows the considered closed-loop system where $G(s)$ represents the power system and $H(s)$ the FACTS POD controller. The POD controller consists of an amplification block, a wash-out and low-pass filters and $m_{c}$ stages of lead-lag blocks as depicted in Fig.4. The transfer function $H(s)$, of the POD controller is given by Equation (15) $K$ is a positive constant gain and $H_{1}(s)$ is the transfer function of the wash-out and lead- lag blocks. $T_{m}$ is a measurement time constants and $T_{w}$ is the washout time constant. $T_{\text {lead }}$ and $T_{l a g}$ are the lead and lag time constant respectively.

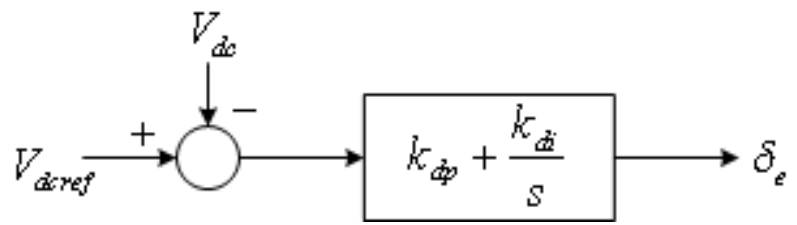

Fig.2 PI-type DC Voltage regulator

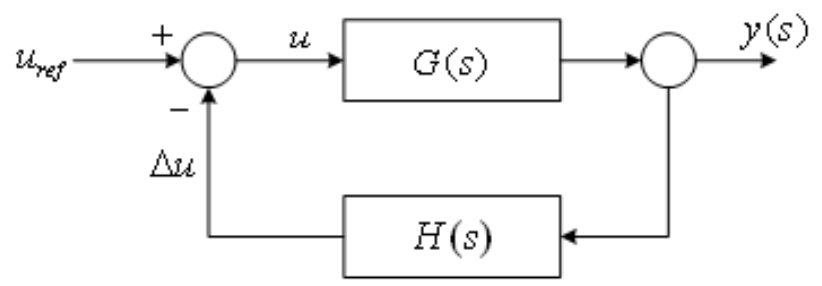

Fig.3. Closed loop system with POD controller

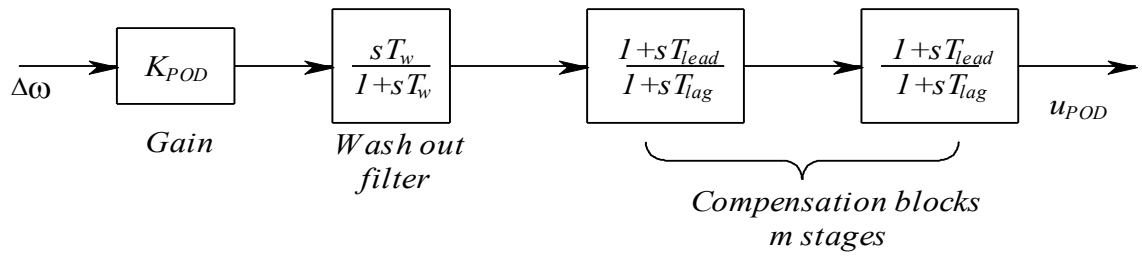

Fig.4. POD controller structure

$$
H(s)=K \frac{1}{1+s T_{m}} \frac{s T_{w}}{1+s T_{w}}\left[\frac{1+s T_{\text {lead }}}{1+s T_{\text {lag }}}\right]^{m_{c}}=K H_{1}(s)
$$

Changes of an Eigen value $\lambda_{i}$ can be described by Equation (16). The objective of the FACTS damping controller is to improve the damping ratio of the selected oscillation mode $i$. Therefore, $\Delta \lambda_{i}$ must be a real negative value in order to move the real part of the Eigen value $\lambda_{i}$ to the left half complex plane.

$$
\nabla \lambda_{i}=R_{i} K H_{1}\left(\lambda_{i}\right)
$$

From Equation (16), it can be clearly seen that with the same gain of the feedback loop, a larger residue will result in a larger change of the corresponding oscillatory mode. Therefore the best feedback signal for the FACTS damping controller is the one with the largest residue for the considered mode of oscillation.

\section{A. GA-Based POD Controller Design}

The parameters of the POD controller are designed based on the genetic algorithm. Before proceeding with GA approach, the suitable coding and fitness function should be chosen. In this study, the parameters $k_{P O D}$, $T_{1}$ and $T_{2}$ for POD controller are expressed in term of string consisting of 0 and 1 by binary code. For our optimization, the following fitness function is proposed. 


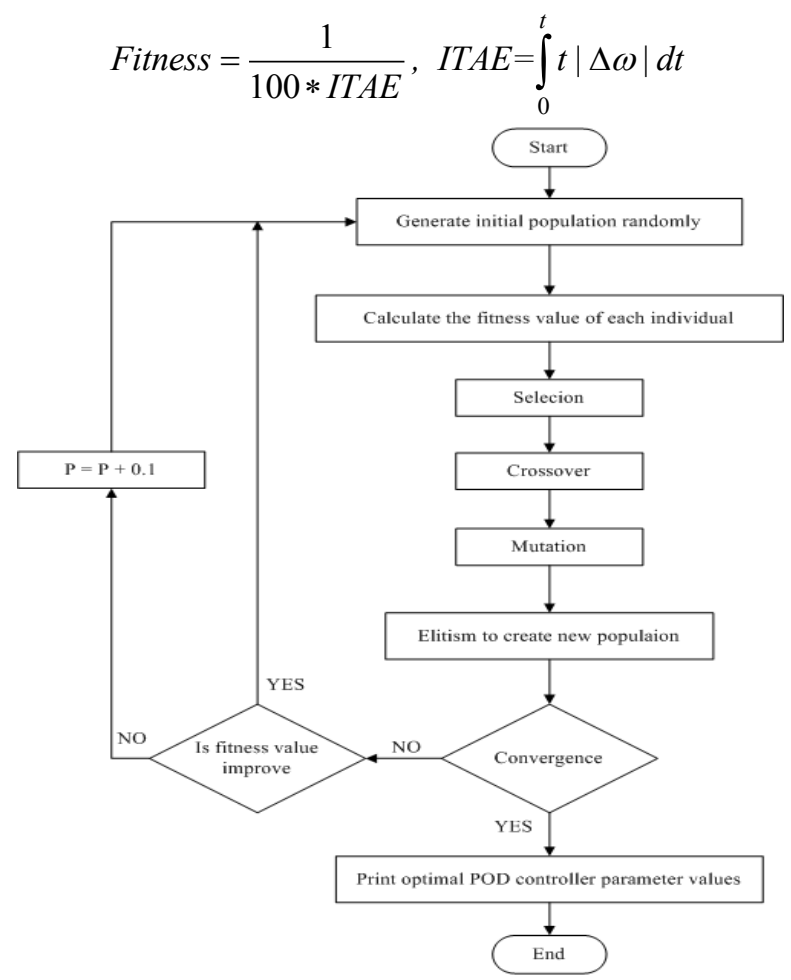

Fig.5. Genetic algorithm flowchart for optimization of POD parameters

For acquiring better performance, number of generation, population size, crosser over rate and mutation rate is chosen 20,10, 0.97 and 0.08 respectively. The proposed flowchart of the genetic algorithm is shown in Fig.5.

\section{Simulation Results}

Different comparative cases are considered in this section to examine the robustness of the proposed GAPOD \& GA DC-voltage regulator in comparison with PSOMSF DC-voltage regulator. The various loading conditions are given in below Table. 1 .

\section{TABLE I. OPERATING CONDITIONS}

\begin{tabular}{|l|c|c|c|}
\hline Operating Points & $P_{e}$ & $Q_{e}$ & $V_{t}$ \\
\hline Nominal Load (operating point 1) & 0.8 & 0.15 & 1.032 \\
\hline Heavy Load(operating point 4) & 1.1 & 0.28 & 1.032 \\
\hline Very heavy Load(operating point 7) & 1.15 & 0.3 & 1.032 \\
\hline
\end{tabular}

The proposed systems simulated with a step disturbance of $0.1 \mathrm{pu}$ at various operating conditions and the results are shown in figures from Fig.6.(a) to Fig.8.(d). With these Figures we concluded that GA POD controller with GA DC-voltage regulator provides effective damping compared with PSOMSF DC-voltage regulator at various operating points.

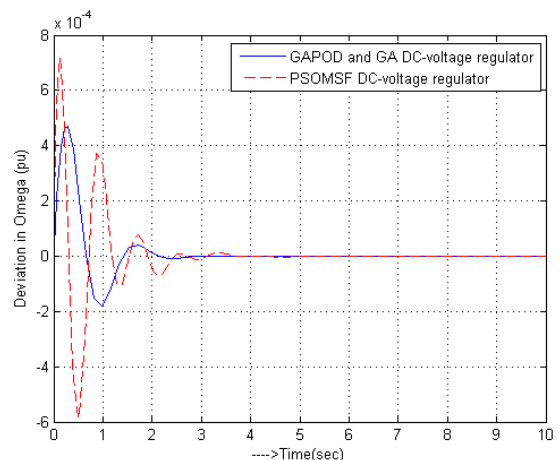

Fig.6. (a) Time response of $\Delta \omega$ with PSOMSF DC- voltage regulator and GA POD and GA DC- voltage regulator at operating point 1 


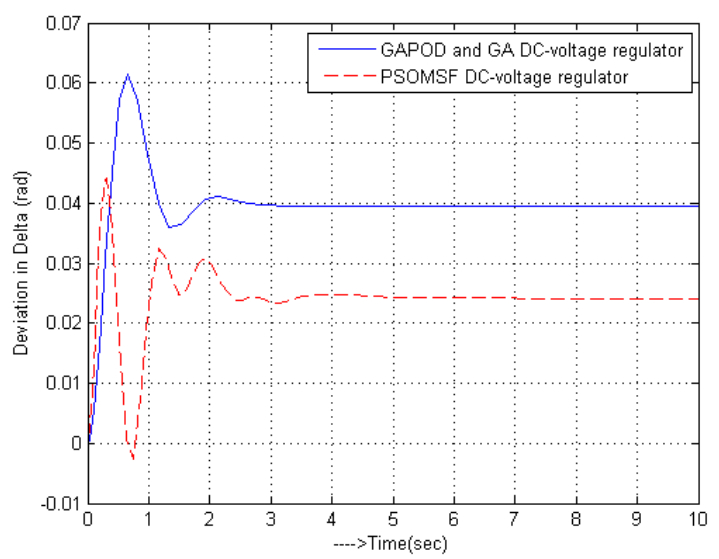

Fig.6 (b) Time response of $\Delta \delta$ with PSOMSF DC- voltage regulator and GA POD and GA DC- voltage regulator at operating point 1

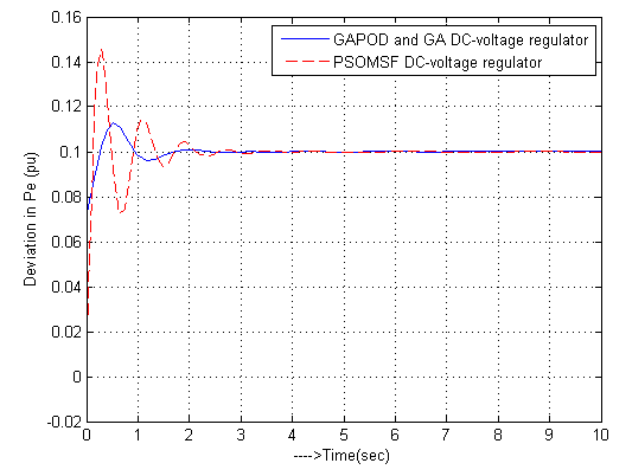

Fig.6 (c) Time response of $\Delta P_{e}$ with PSOMSF DC- voltage regulator and GA POD and GA DC- voltage regulator at operating point 1

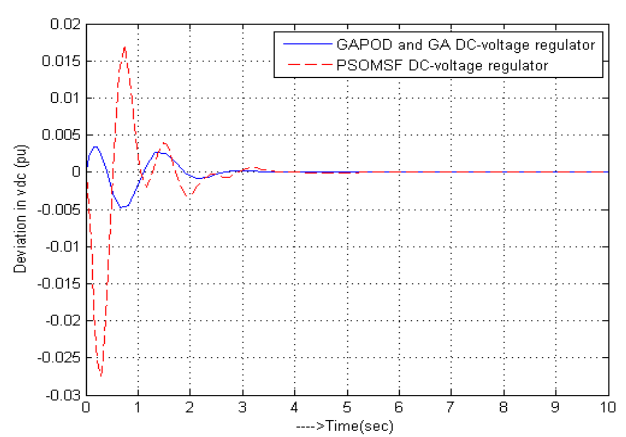

Fig.6 (d) Time response of $\Delta V_{d c}$ with PSOMSF DC- voltage regulator and GA POD and GA DC- voltage regulator at operating point 1

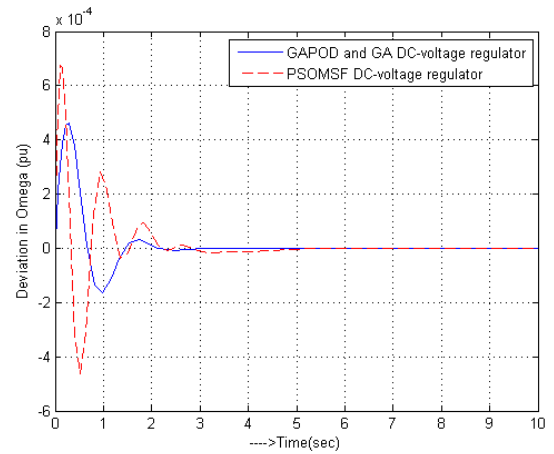

Fig.7 (a) Time response of $\Delta \omega$ with PSOMSF DC- voltage regulator and GA POD and GA DC- voltage regulator at operating point 4 


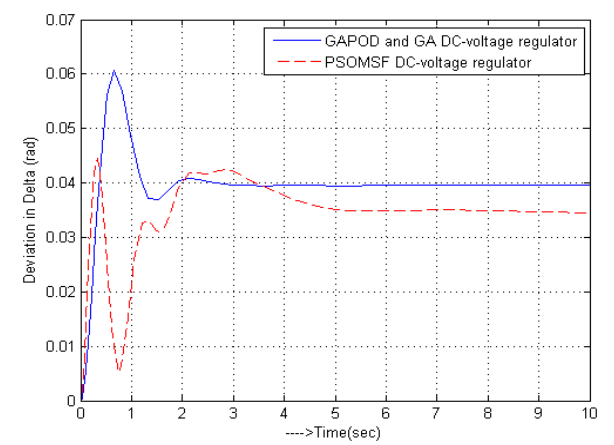

Fig.7 (b) Time response of $\Delta \delta$ with PSOMSF DC- voltage regulator and GA POD and GA DC- voltage regulator at operating point 4

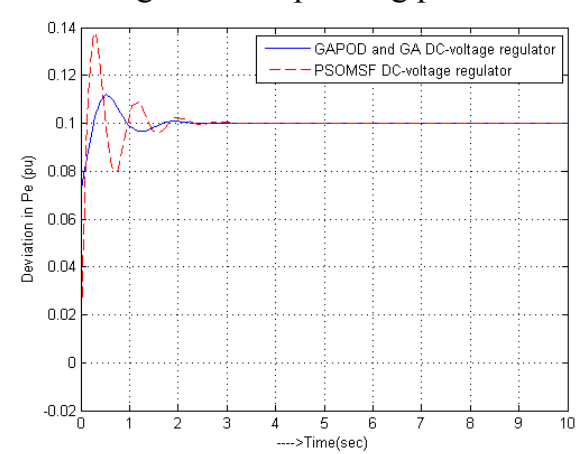

Fig.7 (c) Time response of $\Delta P_{e}$ with PSOMSF DC- voltage regulator and GA POD and GA DC- voltage regulator at operating point 4

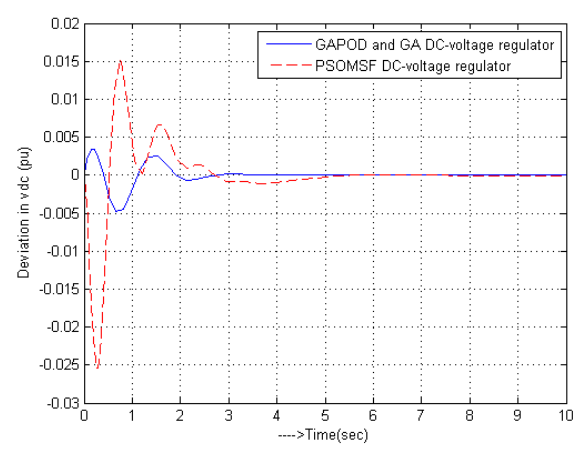

Fig.7 (d) Time response of $\Delta V_{d c}$ with PSOMSF DC- voltage regulator and GA POD and GA DC- voltage regulator at operating point 4

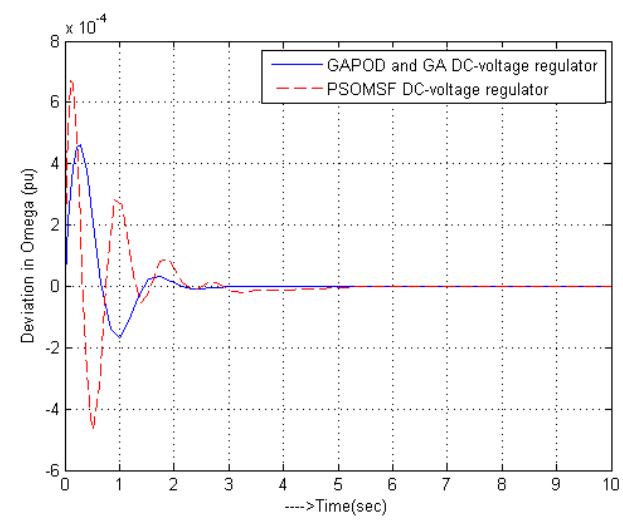

Fig.8 (a) Time response of $\Delta \omega$ with PSOMSF DC- voltage regulator and GA POD and GA DC- voltage regulator at operating point 7 


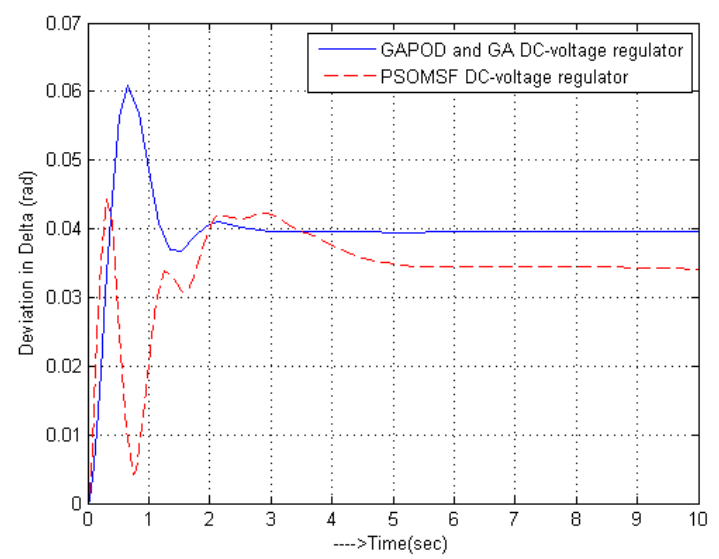

Fig.8 (b) Time response of $\Delta \delta$ with PSOMSF DC- voltage regulator and GA POD and GA DC-voltage regulator at operating point 7

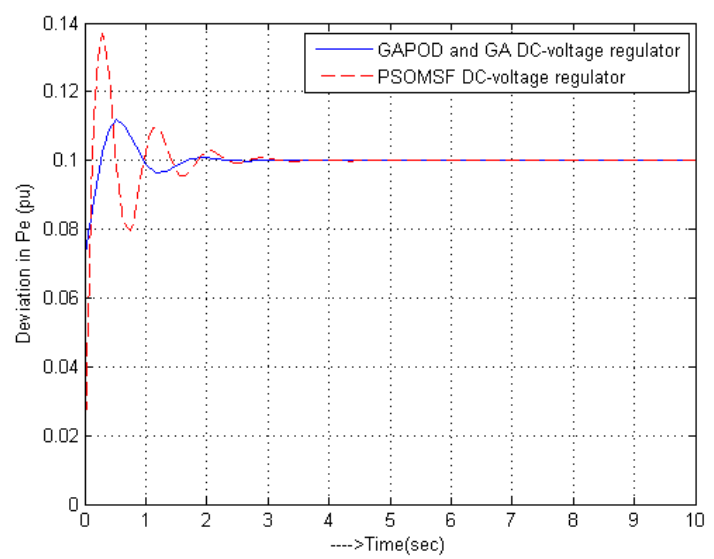

Fig.8 (c) Time response of $\Delta P_{e}$ with PSOMSF DC- voltage regulator and GA POD and GA DC- voltage regulator at operating point 7

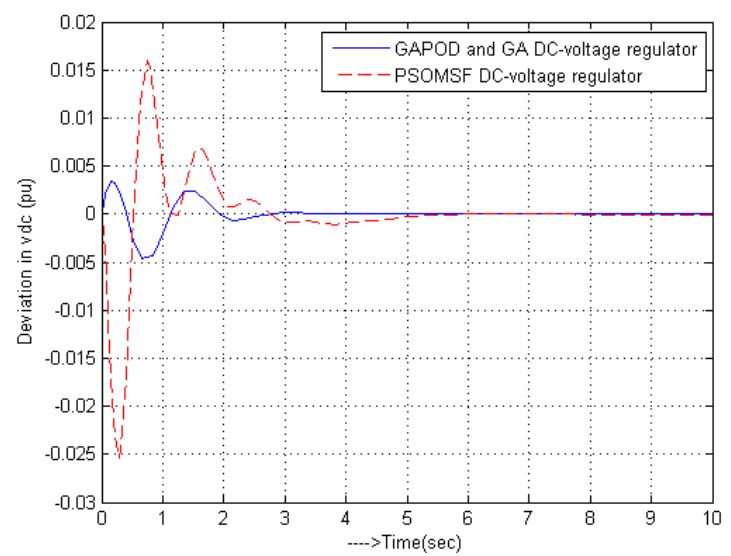

Fig.8 (d) Time response of $\Delta V_{d c}$ with PSOMSF DC- voltage regulator and GAPOD and GADC- voltage regulator at operating point 7

\section{CONCLUSION}

In this paper GAPOD and GADC-voltage regulator is proposed for UPFC for damping low frequency oscillations. The effectiveness of the proposed controller has been tested on a SMIB(double-line) power system in comparison to the PSOMSF DC-voltage regulator under different operating conditions. The result of evaluation shows that the oscillations of synchronous machines can be effectively and quickly damped for power systems with the proposed controller. 


\section{References}

[1] L. Gyugyi, Unified power-flow control concept for flexible ac transmission systems, IEE Proc. On Generation, Transmission and Distribution, Vol. 139 No. 4, 1992, pp. 323-31.

[2] N. Tambey, M.L. Kothari, Damping of power system oscillations with unified power flow controller (UPFC), IEE Proc. On Generation, Transmission and Distribution, Vol. 150, No. 2, 2003; pp. 129-40.

[3] P.K. Dash, S. Mishra, G. Panda, A radial basis function neural network controller for UPFC, EEE Trans. On Power Systems, Vol. 15, No. 4, 2000, pp. 1293-9.

[4] M. Vilathgamuwa, X. Zhu, S.S. Choi, A robust control method to improve the performance of a unified power flow controller, Electric Power Systems Research, Vol. 55, 2000, pp.103-11.

[5] B C. Pal, Robust damping of interarea oscillations with unified power flow controller, IEE Proc. On Generation, Transmission and Distribution, Vol. 149, No. 6, 2002, pp. 733-8.

[6] J.-C. Seo, S.-I. Moon, J.-K. Park, J.-W. Choe, Design of a robust UPFC controller for enhancing the small signal stability in the multi-machine power systems, Proc. of the IEEE PES Winter Meeting, Vol. 3, 28 January-1 February, 2001, pp. 1197-202.

[7] L-X. Wang, A course in fuzzy systems and control, NJ: Prentice Hall; 1997.

[8] M.E. El-Hawary, Electric power applications of fuzzy systems, New York: IEEE Press; 1998.

[9] K.L Lo., Y.J. Lin, Strategy for the control of multiple series compensators in the enhancement of interconnected power system stability, IEE Proc. On Generation, Transmission and Distribution, Vol. 146, No. 2, 1999, pp. 149-158.

[10] A. Kazemi, M. Vakili Sohrforouzani, Power system damping controlled facts devices, Electrical Power and Energy Systems, Vol. 28, 2006, pp. 349-357.

[11] P.K. Dash, S. Mishra, G. Panda, Damping multimodal power system oscillation using hybrid fuzzy controller for series connected FACTS devices, IEEE Trans. on Power Systems, Vol. 15, No. 4, 2000, pp. 1360- 1366.

[12] L. Khon, K. L. Lo., Hybrid micro-GA based FLCs for TCSC and UPFC in a multi machine environment, Electric Power Systems Research, Vol. 76, 2006, pp. 832-843

[13] T.K. Mok, H. Liu, Y. Ni, F. F. Wu, R. Hui, Tuning the fuzzy damping controller for UPFC through genetic algorithm with comparison to the gradient descent training, Electric Power and Energy Systems, Vol. 27, 2005, pp. 275-283.

[14] Wang HF. Damping function of unified power flow controller. IEE Proc Gen Transm Dist 1999;146(1):81-7.

\section{Authors' information}

D. Obulesu received the B.E. degree in EEE from STJ Institute of Technology, Ranebennur, Karnataka, India from Karnataka University and M.Tech. in Power Electronics and Drives from Dr. M.G.R. University in the year 2000 and 2005 respectively. He has got a teaching experience of nearly 09 years. Currently, he is working as Associate Professor in VEMU Institute of Technology P.kothakota, Chittoor Dist, Andhra Pradesh, India in the Dept. of Electrical \& Electronics Engg. \& simultaneously doing his Ph.D. (Research Scholar) in Electrical \& Electronics Engg. from the prestigious Jawaharlal Nehru Technological University (JNTU), Hyderabad, Andhra Pradesh, India. He has published a number of research papers in various national \& international journals \& conferences. His area of interests are neural networks, fuzzy logic, artificial intelligence, power electronics, Matlab, FACTS, etc.

Dr. S. F. Kodad received the B.E. degree in EEE from Karnataka University and the M.Tech. degree in Energy Systems Engg. from JNTU, Hyderabad, India in the year 1992. He received his Ph.D. degree in Electrical Engg. from JNTU, Hyderabad, India in the year 2004. He has got a teaching experience of nearly 25 years. Currently, he is working as Principal in Krishna Murthy Institute of Technology and Engineering, Hyderabad, Andhra Pradesh, India. He has published a number of papers in various national \& international journals \& conferences $\&$ done a number of in-house $\&$ industry projects. He has also presented a number of guest lectures and various seminars and participated in a number of courses, seminars, workshops, symposiums in the various parts of the country in different institutions and also conducted a few courses. He is also guiding a number of Ph.D. students. His area of interests are neural networks, fuzzy logic, power electronics, power systems, artificial intelligence, Matlab, Renewable energy sources, etc.

Dr. B. V. Sankar Ram received the B.E. degree in Electrical Engg. from Osmania University \& M.E. degree in Power Systems from Osmania University, Hyderabad, Andhra Pradesh, India. He received his Ph.D. degree in Electrical Engg. from JNTU, Hyderabad, India. He has got a teaching experience of more than 28 years. Currently, he is working as Director, Admission in JNTU College of Engg. Hyderabad, India. He has published a number of papers in various national \& international journals \& conferences \& done a number of in-house \& industry projects. He is also guiding a number of research scholars in various topics of engg. He has specialized in power systems. His research interests include power system reliability and Flexible AC Transmission systems, power electronics \& it is applications. 